\title{
CONSTRUCCIONES EN YESO PROYECTADO SOBRE VEGETALES
}

\author{
(GYPSUM PROJECTED ON WILD VEGETABLES)
}

\author{
Hubert Martinez, Arquitecto \\ Traducción y adaptación: M.a Jesús Guinea Diaz, Arquitecto, \\ IETcc/CSIC
}

$127-16$

\section{RESUMEN}

Procedimiento de construcción desarrollado al comienzo de 1981 en el Senegal, teniendo como objetivo la valoración de las fuentes naturales y humanas, especialmente los vegetales en estado silvestre $y$ el fosfoyeso, residuo de la industria quimica del Senegal. La introducción de la proyección mecánica permite aumentar sensiblemente el rendimiento del trabajo y mejorar la calidad. La proyección reduce el número de oficios que intervienen en la obra por la supresión de enlucidos, sistemas de estanquidad y pinturas sobre la gruesa obra y simplifica la colocación de las instalaciones técnicas y de carpintería.

La adquisición del material de proyección, necesitando un capital relativamente modesto, se encuentra al alcance de pequeñas y medianas empresas que deseen especializarse en los trabajos de yeso. La rentabilidad financiera del procedimiento está asegurada para un precio del yeso de hasta el $180 \%$ del precio del cemento.

\section{SUMMARY}

A construction method developed in early 1981 in Senegal, whose main objective is the evaluation of natural and human sources, specially those of wild vegetables and the phosphogypsum (a waste of chemical industry in Senegal). The introduction of the mechanical projection allows a noticeably growth in work performances and an improvement of the quality. The projection reduces de number of trades intervening in the work, doing away with plasters, watertightness systems and paintings, and simplifying the setting of technical installations and of carpentry.

The acquisition of this projecting material is not very expensive, so, it is easy for small and medium enterprises to reach an expertness in gypsum work. Its finantial rentability is assured for a prize of the gypsum of $180 \%$ of the prize of the cement.

\section{ANTECEDENTE CONSTRUCTIVO}

Considerando la proyección del yeso sobre un soporte vegetal como un procedimiento de construcción que permite realizar obras tridimensionales y monolíticas, es posible hablar de antecedentes en Europa y en el Senegal.

En Europa, son realizados en proyección sobre rejilla metálica; en Senegal, por el contrario, en ramajes, hierbas, tierra y recientemente también utilizando cemento.

En Senegal existen diferentes formas y técnicas para construcciones tradicionales a base de vegetales. Globalmente se puede clasificar en dos grupos:

- construcciones de nómadas,

- construcciones de tribus sedentarias.

En el presente marco solo el aspecto constructivo tiene interés, el aspecto arquitectónico puede ser olvidado.
Las chozas de los nómadas varian en la forma, pero el carácter temporal les es común, por el hecho de los desplazamientos frecuentes de sus habitantes. En consecuencia, sus construcciones están limitadas a la utilización de ramas, ramitas, hierbas y hojas (desgraciadamente desfiguradas hoy a causa del material utilizado). Pero las estructuras espaciales de los nómadas son las más bellas y dignas de ser valoradas, aumentando su longevidad y terminación para mejorar la higiene y su resistencia al fuego.

Las construcciones de las tribus sedentarias se distinguen principalmente por la utilización de materiales pesados en combinación con los vegetales: la tierra y ramas de otras especies de árboles o de bambúes disponibles. Están desarrolladas técnicas regionales que, por una parte, se basan en el relleno de los muros con bolas de tierra o argamasa de barro y paja sobre un entramado de ramas (Casamance) y, por otro lado, sobre un trenzado de cañas de bambúes partidas (región de Kaolack). Estas últimas construcciones tienen un replanteo octogonal; la cubierta 


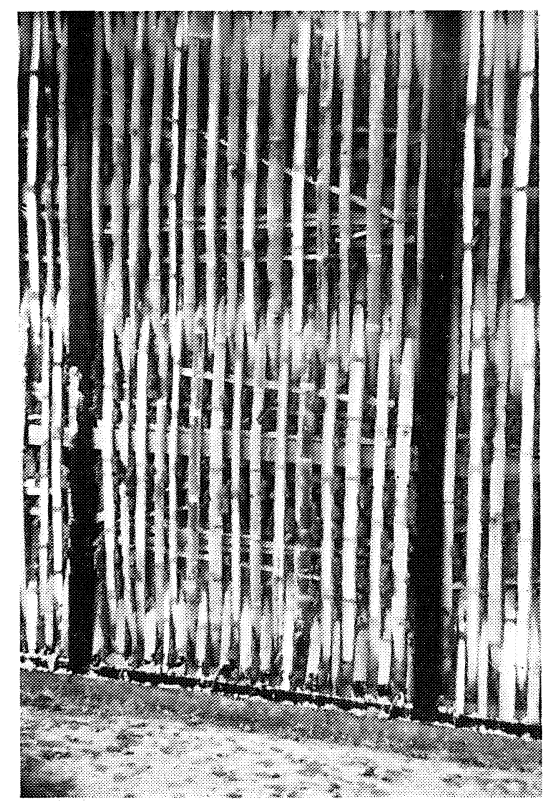

Soporte vegetal para yeso.

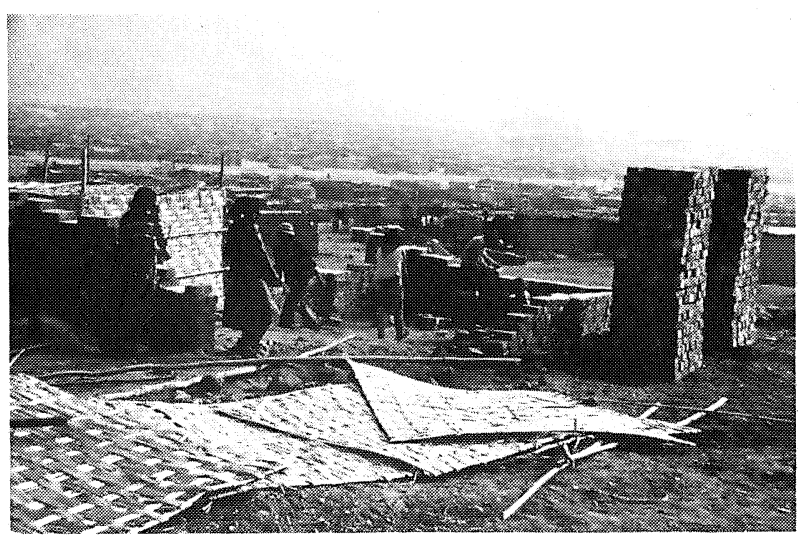

La construcción de los soportes es una tradicion artesana.

a dos aguas; una concepción muy europea, desgraciadamente carente de la diagonal necesaria para construir el triángulo estáticamente indeformable.

Lo importante es el enlucido del trenzado sobre los dos lados por una capa de tierra, hoy generalmente reemplazado por enlucido del cemento.

Tenemos como antecedente constructivo a valorar:

- las estructuras espaciales nómadas trabajando a compresión,

- el enlucido con tierra de las cañas trenzadas de la región de Kaolack.

En Europa, las formas espaciales o láminas en yeso sobre entramado de pequeña sección de $1 \mathrm{~cm}^{2}$ son corrientes desde hace unos cientos de años, pero sin tener una función estática portan- te. Su composición se ha podido comprobar en los escombros de la guerra, en 1945 y años siguientes.

Desde hace una docena de años la proyección de los morteros de hormigón toman más y más importancia partiendo de obras de ingenieria civil (túnel), pasando por proyecciones contra el fuego, el agua y los enlucidos ordinarios, con objeto de orientarse hacia la construcción de viviendas.

Los soportes están constituidos por armaduras de mallas galvanizadas, telas metálicas galvanizadas o metal estirado y revestido de enlucido proyectado sobre base de cemento, yeso y de resinas. Hoy, se da cada vez más la utilización de productos quimicos, en particular el poliestireno que es utilizado como alma llena con armaduras para servir de pantalla de proyección durante la construcción y de aislamiento térmico más tarde.

Tomamos como antecedente el material de proyección mecánica; por el contrario, los productos industriales son desechados para una utilización en Africa.

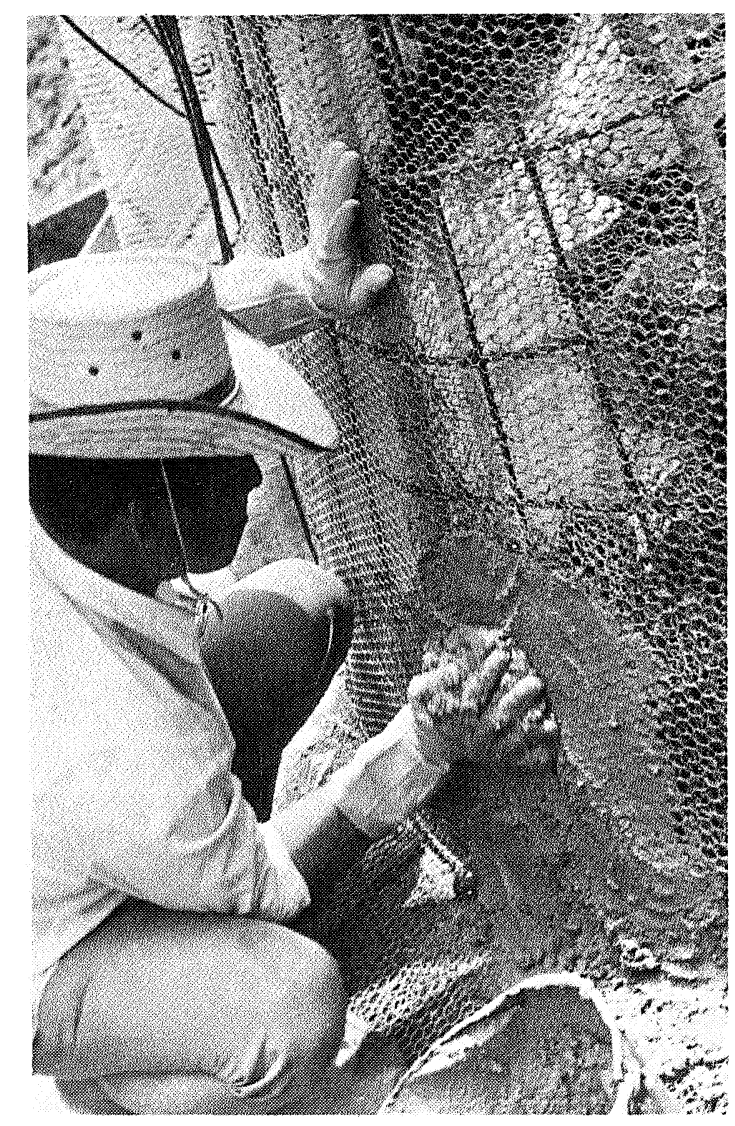

Con frecuencia la malla sustituye al soporte vegetal. La aplicacion sigue siendo manual. 


\section{JUSTIFICACION ECONOMICA}

La justificación económica está basada en el modelo de la movilización de recursos. Estos últimos comprenden los recursos naturales por una parte y los recursos humanos por otra. Para llegar a una movilización reciproca es preciso pasar por etapas sucesivas y comenzar por la simple utilización de recursos naturales. A corto plazo, y especialmente en el sector de la vivien$\mathrm{da}$, la distinción entre recursos renovables y no renovables no tiene gran importancia, puede ser reemplazada por la simple diferenciación: abundante o rara, y disponible sobre el lugar o no.

El yeso es un recurso natural abundante en la región del norte de Nonakchott. Se encuentra en la superficie, en estado polvoriento.

Su transformación en yeso, por simple cocción entre 100 y 1400 , requiere menos energia en combustible que la fabricación del cemento. El yeso es pues una fuente natural doblemente rentable para Mauritania:

- rentabilidad económica dada para el estado debido a que el cemento en venta, en Mauritania, es de importación y solamente puesto en seco en Mauritania;

- la rentabilidad financiera deberá estar asegurada tanto para el productor como para el consumidor.

En Senegal, la industria quimica sobre base de fosfato origina un residuo, el fosfoyeso, que normalmente es tirado al mar.

Para recuperar el fosfoyeso contenido en las aguas usadas de la fábrica, la producción del ácido fosfórico es interrumpida para poder filtrar las aguas. Hay pues interrupción de producción; es decir, que el residuo no se dispone gratuitamente y su transformación en yeso todavia menos. Su transformación pasa por diversas etapas que consumen todas las energias. La fábrica de yeso y la fábrica de cemento se acusan mutuamente de consumir más energia por tonelada de producto acabado.

La rentabilidad económica y financiera del yeso químico del Senegal es menos evidente que la del yeso natural. La misma situación en Francia habria tenido como consecuencia el cierre de fábricas de yeso quimico.

Por tanto, la fábrica de producción de yeso del Senegal, perteneciendo hoy a las Industrias Químicas del Senegal funciona desde su construcción, en 1976, con una pequeña cuota de utilización que iba de 10 a $25 \%$, que excluye normalmente la rentabilidad financiera.

El fabricante tiene en cuenta su política de precios; lo principal es el precio de venta de pro- ductos competitivos. Así, el precio para los diferentes productos y calidades de yeso varia entre 26 y $245 \mathrm{~F} / \mathrm{kg}$ y es independiente del valor añadido por la mano de obra. Como prueba, es suficiente citar un ejemplo: el yeso para moldear ordinario es vendido por saco al precio de $58.500 \mathrm{~F} / \mathrm{t}$ y los paneles huecos de $15 \mathrm{~cm}$ de espesor, fabricados con esta calidad de yeso, son vendidos a $26 \mathrm{~F} / \mathrm{kg}$.

Para tener éxito en el plan financiero, el fabricante contrata las construcciones del consumidor que no conoce las cualidades del yeso, pero que se contenta con la simple utilización del yeso como polvos a colmatar, con el fin de enlucir por una parte y como producto moldeado para los falsos techos. El fabricante encuentra su rentabilidad en estos productos, que son comercializados entre 183 y $245 \mathrm{~F} / \mathrm{kg}$, bien que los precios estén fijados por pieza y no por kilogramos. La simple utilización del yeso por una mano de obra poco cualificada para la puesta en obra, no desvia un factor de desarrollo.

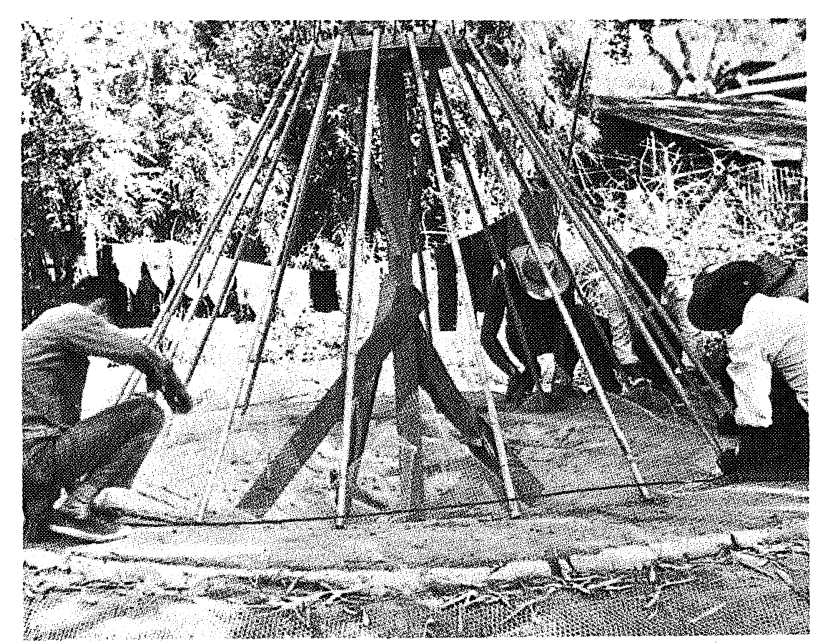

Actualización de técnicas ancestrales.

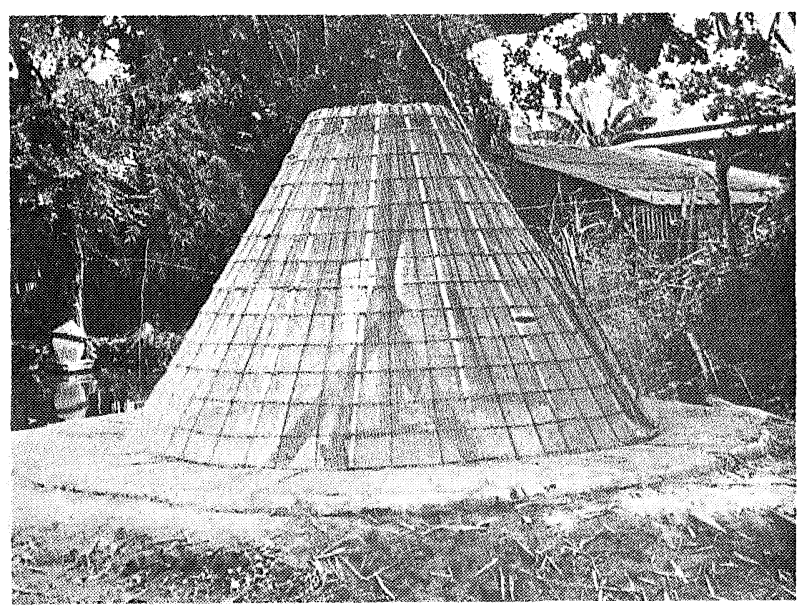

Soporte preparado para la aplicación del yeso. 
Es preciso pasar por una fase de valoración del yeso. Esta fase ha sido llevada a cabo por las acciones del REXCCOP, principalmente por el concurso de viviendas en yeso, pero este concurso, sin realización de los proyectos presentados, no ha podido aportar "el conocimiento". Para provocar el factor catalizador, es preciso que la revalorización de los recursos naturales sean acompañados de los recursos humanos. En términos claros, para poder valorar un material, es necesario igualmente cualificar a su utilizador. Por este motivo, no se preconiza la tendencia dominante de utilizar el yeso como sustitutivo del cemento y de los productos del cemento. Es necesario sacar beneficio de las ventajas del yeso en relación al cemento, cualificar la mano de obra para puesta en obra de estas ventajas y encontrar en consecuencia inevitable una nueva expresión arquitectónica. Solamente este aprovechamiento permite participar en el proceso de movilización de los recursos naturales y humanos $y$, en consecuencia, en el desarrollo socioeconómico.

Para cercar el ámbito en el que la utilización del yeso se sitúa con una rentabilidad directa, no es suficiente comparar simplemente el precio de un metro cuadrado de superficie habitable, es preciso comparar el coste de los elementos de construcción terminados, realizados en yeso (aqui el término "coste" está bien utilizado en oposición al término "precio"). Los dos elementos más importantes en la construcción de una vivienda, que pueda estar realizada en yeso, son "el muro" y «la cubierta».

Si es posible calcular la cantidad exacta de diferentes materiales que entran en la fabricación de un elemento, es más dificil determinar el número de trabajo necesario para la fabricación del mismo. Es imposible expresar el valor de dos componentes (materiales y mano de obra) sin tener en cuenta el medio, es decir, el lugar de la construcción.

En la región de Dakar, se basan en la comparación de cuatro tipos de elementos «muro».

1. Panel de muro de $3 \mathrm{~m}$ de altura y $4 \mathrm{~m}$ de longitud, compuesto como sigue:

- Bloques huecos artesanales de $15 \mathrm{~cm}$ de espesor, realizados con arena y cemento.

- Apeos horizontales y verticales a la italiana, de sección $15 \times 20 \mathrm{~cm}$.

- Enlucido de cemento de $2 \mathrm{~cm}$ sobre las dos caras del muro.

- Pintura plástica sobre las dos caras.

2. Panel de muro de las mismas dimensiones $y$ composición; bloques fabricados siguiendo calidad controlada.
3. Panel de muros de las mismas dimensiones compuesto como sigue:

- Placas de yeso colocadas, de $15 \mathrm{~cm}$ de espesor.

- Enlucido hidrófugo en yeso sobre cara exterior.

- Raspado sobre superficie interior.

- Pintura plástica sobre cara interior.

4. Panel de muro de las mismas dimensiones compuesto como sigue:

- Armadura entramada soldada en hierro con hormigón $\varnothing 6$ a $10 \mathrm{~mm}\left(2 \mathrm{~kg} / \mathrm{m}^{2}\right)$.

- Soporte con hierbas trenzadas o similar.

- Yeso proyectado de $3 \mathrm{~cm}$ de espesor sobre las dos caras, en el cual $1,5 \mathrm{~cm}$ es hidrofugado del lado exterior.

En cuanto a la cubierta es necesario proceder de la misma forma:

1. Cubierta de placas onduladas compuesta:

- Perfiles metálicos IPN-80 $\left(70 \mathrm{~kg} / \mathrm{m}^{2}\right)$.

- Cobertura en amianto-cemento.

- Pintura sobre armazón.

- Falso techo suspendido sobre armazón.

- Pintura sobre techo.

2. Cubierta maciza en B.A. (hormigón armado) compuesta:

- Losa de hormigón armado de $10 \mathrm{~cm}$ de espesor.

- Forma, en pendiente.

- Estanquidad multicapa autoprotegida.

- Enlucido de techo.

- Pintura de techo.

3. Cubierta de yeso sobre molde, compuesta:

- Cúpula de $5 \mathrm{~cm}$ de espesor en yeso hidrófugo proyectado sobre molde en poliestireno.

- Armadura en tela de fibra de vidrio.

- Raspado interior.

4. Cubierta en yeso proyectado sobre soporte vegetal, compuesto:

- Rigidizador de entramado con tirante en hierro con hormigón de $\varnothing 6$ a $10 \mathrm{~mm}$ (2 $\mathrm{kg} / \mathrm{m}^{2}$ ).

- Soporte con hierba trenzada o similar.

- Yeso proyectado de $3 \mathrm{~cm}$ de espesor sobre las dos caras, del cual $1,5 \mathrm{~cm}$ en yeso hidrófugo en el lado exterior. 
La comparación de costes por metro cuadrado, sea de muro, sea de cobertura, permite una primera elección. Actualmente los costes de los elementos "muros» y "cubierta", en yeso, son inferiores a los de otros elementos comparados.

Siempre para evaluar la incidencia de los elementos componentes de un edificio, es imprescindible relacionar sus costes sobre la superficie útil, es decir, la superficie de suelo entre muros.

En efecto, cuando se considera la superficie habitable de un local de $3 \times 4 \mathrm{~m}$ es necesario deducir la envoltura exterior, es decir, los muros. En el caso de muro de $15 \mathrm{~cm}$ más $2 \times 2 \mathrm{~cm}$ de enlucido, la superficie ocupada por esta envoltura es de $20 \%$ de la superficie bruta. Para el caso de un cerramiento con yeso proyectado sobre vegetales de $6 \mathrm{~cm}$ de espesor, la superficie "perdida" es inferior al $10 \%$. En consecuencia, hay una ganancia del $10 \%$ aproximadamente de superficie útil, donde se da una rentabilidad financiera igual si el precio unitario es más elevado para un tabique proyectado sobre vegetales.

En cuanto a la cubierta, la valoración es más compleja, pues es necesario tener en cuenta dos criterios:

- la relación entre superficie desarrollada de cubierta y superficie útil,

- la altura libre del local construido.

En consecuencia, la comparación debe hacerse sobre la base del volumen útil.

En efecto, el volumen útil depende de la forma de la cubierta y del falso techo eventual. Una cubierta plana necesita una altura de muros de 2,8 $\mathrm{m}$ aproximadamente. Por el contrario, bajo una cubierta en forma de cúpula o de bóveda, la altura de los muros puede ser rebajada $2,20 \mathrm{~m}$, aproximadamente la altura de paso libre.

Si la relación superficie desarrollada / superficie útil es desfavorable para las cúpulas y bóvedas, especialmente para inclinación reducida, esta relación se mejora con la inclinación creciente. Por ejemplo para las bóvedas, esta relación baja de 1,66 para la inclinación de 3,2 $\mathrm{m}$ a 1,27 para las de 7,2 m. En consecuencia, el coste disminuye «inversamente proporcional» a la inclinación.

Por el contrario, las cúpulas y bóvedas permiten rebajar la altura de los muros de 2,8 a 2,2 m, con una reducción del $22 \%$ del coste.

En el contexto económico del Senegal, región de Dakar, las construcciones de yeso son pues rentables, tanto sobre la base de un precio de yeso para proyectar de $81.900 \mathrm{~F} / \mathrm{t}$, como $101.400 \mathrm{~F} / \mathrm{t}$ para la calidad hidrófugo, en relación a un precio de cemento de $44.448 \mathrm{~F} / \mathrm{t}$ (precios oficiales en el mes de febrero 1986).
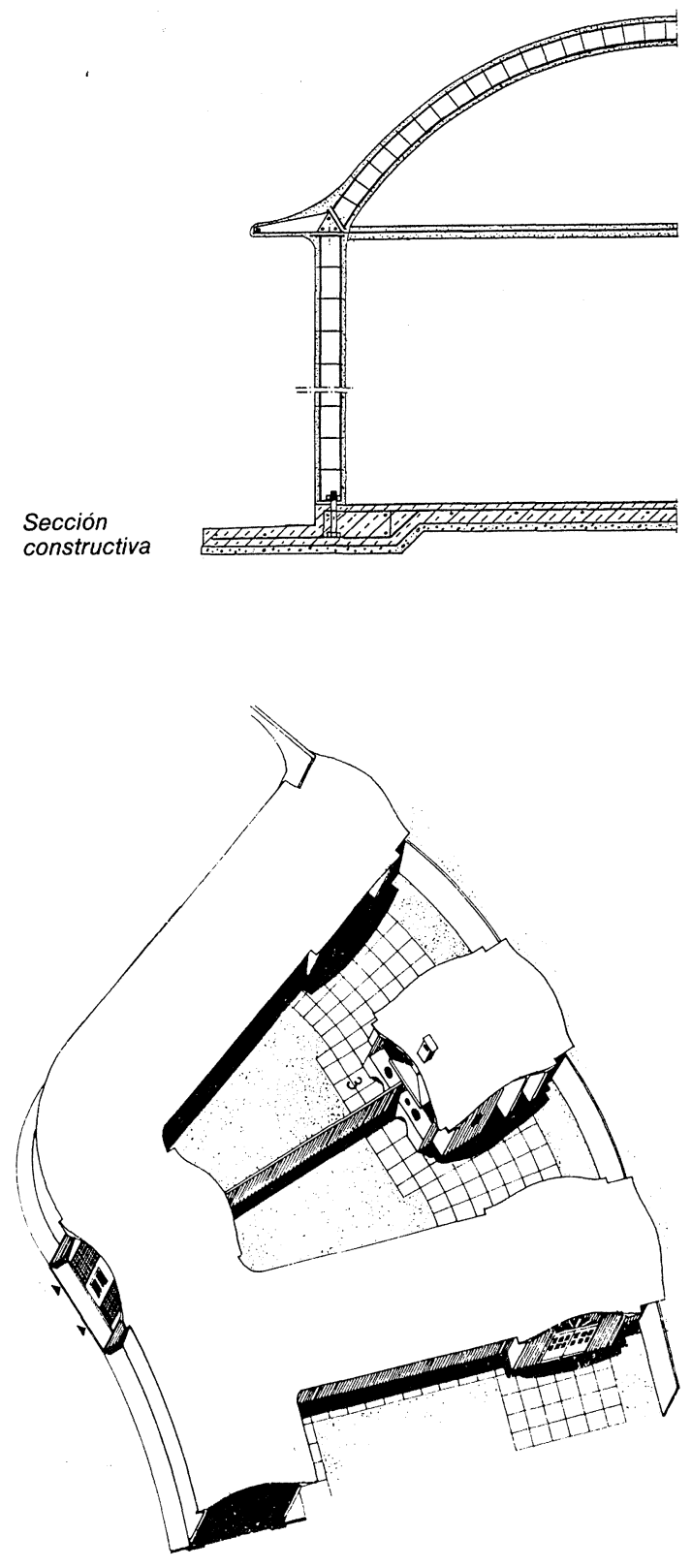

Perspectiva del edificio 


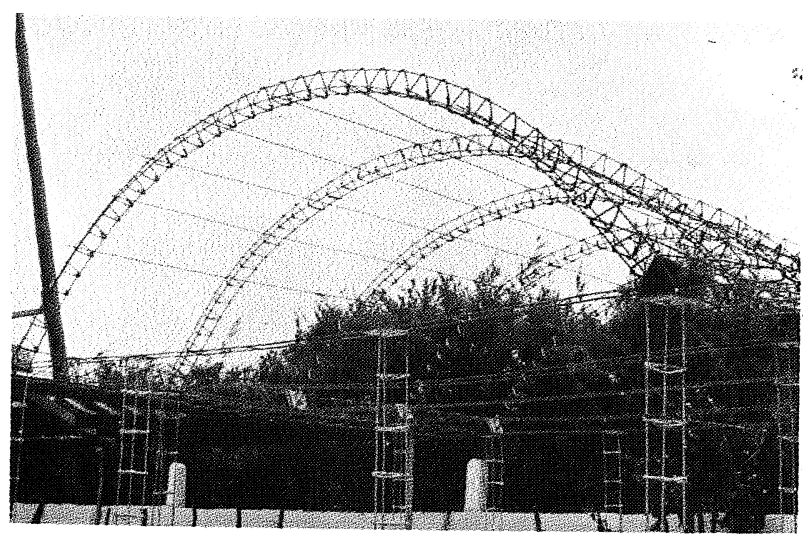

Las cimbras metálicas constituyen la estructura.

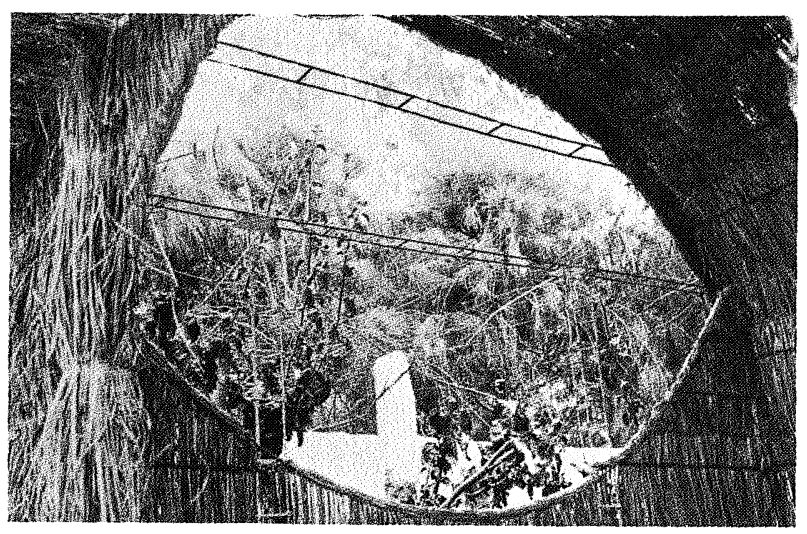

Estado del soporte antes de la aplicación del yeso.

\section{INVESTIGACION TEORICA}

La utilización tradicional de recursos locales no conlleva necesariamente el desarrollo económico ni técnico. Al contrario, se constata generalmente una regresión y una sustitución por modelos importados. Así la autoconstrucción se caracteriza por una disminución del trabajo invertido y por un aumento de los componentes importados. En este contexto, igual los materiales de reciclaje, tal como las planchas de cajas de conserva, los sacos de plástico son considerados como componentes importados, que reemplazan los recursos naturales.

En consecuencia, la investigación teórica relativa a la revalorización de recursos locales debe incluir los dos componentes:

- el perfeccionamiento de recursos naturales (materiales),

- la cualificación de los recursos humanos (mano de obra).

\section{EXPERIMENTACION PRACTICA}

El procedimiento de proyección del yeso sobre soporte vegetal responde a la vez a estos dos criterios.
- El hecho de haber utilizado soportes de hierro con hormigón para las construcciones en medio urbano en lugar de ramas de madera no es una contradicción. La utilización de ramas está reservada para el medio rural, puesto que son disponibles alli. Técnicamente éstas son tambièn válidas bajo condición de limitarse a pequeños diámetros, si no el yeso fisuraria y desprendería.

- La proyección mecánica está igualmente justificada en el medio urbano y está al alcance de pequeñas o medianas empresas que quieren especializarse en este género de trabajos. El procedimiento suprime la intervención de muchos oficios: enlucidor, pintor, etc., pues el edificio no necesita ni enlucido, ni pintura... Una aplicación manual de yeso es posible, pero con la imposibilidad de garantizar una calidad constante de la amasada y de eliminar la puesta en obra del yeso "muerto", en exceso, dentro del plazo antes de la toma. La aplicación manual requiere un soporte más rigido que el realizado con las hierbas silvestres. Las trenzas en tiras partidas combinan perfectamente.

Un problema común en las dos aplicaciones es la necesidad de poder acceder a todo lugar de la obra durante los trabajos antes de la toma del yeso.

Por razón económica es preferible renunciar al andamiaje y reforzar por contrario la estructura de obra, de manera que los obreros puedan subir encima para trabajar como es practicado sobre los edificios tradicionales.

La experiencia de las diferentes obras lleva a preconizar dos soluciones para edificios aislados:

- Reducir la separación de los rigidizadores de la bóveda a la separación de la proyección y desmontar sucesivamente durante los trabajos.

Para los edificios en repetición:

- Trabajar con un pórtico de andamiaje móvil, bajo el cual esté suspendido el soporte vegetal. Después de la toma del yeso, el pórtico es desplazado para la proyección del tramo siguiente.

De forma general, la obra se desenvuelve en 3 fases:

- prefabricación del armazón sea en taller, sea sobre obra,

- realización de la infraestructura en hormigón armado,

- realización de la superestructura en yeso. 
La superestructura está compuesta de 3 elementos estandarizados para permitir la prefabricación y el ensamblaje por bulones:

- poste, viga de apeo horizontal, tensor de bóveda, tirante.

Esta armadura no trabaja solidariamente con el yeso según la teoria del hormigón armado. Lleva los entrenzados vegetales que deben estar empotrados en yeso. Los aceros están igual desolidarizados con el yeso por hierbas. Siempre la rigidización de la estructura está prevista durante la fase de proyección, pues debe soportar la carga del yeso antes de su toma y eventualmente a los obreros trabajando sobre la estructura sin andamiaje.

Sin embargo, la ligereza de los elementos de estructura ( 3 a $4 \mathrm{~kg} / \mathrm{ml}$ ) permite un montaje cómodo en algunas horas, sin utilización de máquina elevadora.

Sobre la estructura montada son fijados los trenzados vegetales, cuyas calidades han sido puestas en obra para comprobar su fiabilidad. La preparación de los vegetales necesita un entrelazado de hierros repartidos de 30 a $50 \mathrm{~cm}$. Su ventaja es la fácil adaptación a toda forma y curva. Pero si la capa de hierbas no está correctamente concebida, se desgarra durante la proyección bajo el peso del yeso fresco.

La preparación con trenzado, formado de tiras partidas y entrelazadas en los dos sentidos, se realiza fácilmente sobre superficies planas 0 curvas. Pero se dan algunos trenzados que por su rigidez, se adaptan mal a las formas curvas en dos planos (cúpula).

La proyección comprende 3 fases sucesivas:

- capa de impregnación teniendo por principio colmatar los huecos y allanar los tallos de hierbas que sobresalen en todas las direccio- nes. Es alisado ligeramente para igualar el soporte;

- capa de enlucido de $2 \mathrm{~cm}$ de espesor, aproximadamente, igualada con la regla o la llana;

- capa de terminación de 0,5 a $1 \mathrm{~cm}$ de espesor cuyo aspecto puede ser elegido libremente entre diversas terminaciones: rústica o gotelé, tirolesa fina o lisa, cortada o alisada. La terminación puede igualmente comprender la colocación del yeso aportado con el agua de amasada. La capa de terminación de las superficies expuestas a la intemperie se realiza con $15 \mathrm{~cm}$ de espesor con yeso hidrófugo.

En la incorporación de un hidrófugo quimico se está investigando la resistencia durante las 4 estaciones después de la construcción de los primeros prototipos.

\section{CONCLUSIONES}

El procedimiento es satisfactorio, el rendimiento práctico, teniendo en cuenta todas las prestaciones es de $2 \mathrm{~m}^{2}$ por hora y equipo compuesto por 6 personas.

Las propiedades mecánicas y físicas son excelentes; el aislamiento térmico de un tabique de $6 \mathrm{~cm}$ corresponde a un muro en hormigón de $24 \mathrm{~cm}$.

La durabilidad observada durante 4 años y el estado de las muestras realizadas in situ son pruebas a considerar para el documento de la garantía decenal.

La rentabilidad financiera está actualmente asegurada a la vez con un precio de yeso de $80 \%$ superior al del cemento.

El procedimiento ha sido aplicado para la construcción de cuatro edificios de $3,2 \mathrm{~m}$ a 7,2 $\mathrm{m}$ de luz libre sin alcanzar los límites técnicos. 